\title{
Crystal and Molecular Structure of N-[2-(6-Methoxy-2-oxo-2H-Chromen-4-yl-Benzofuran-3-yl]- Benzamide
}

\author{
G. Anuradha ${ }^{1}$, G. Vasuki ${ }^{1 *}$, Imtiyaz Ahmed Khan², Manohar V. Kulkarni² \\ ${ }^{1}$ Department of Physics, Kunthavai Naachiar Government Arts College, Thanjavur, India \\ ${ }^{2}$ Department of Chemistry, Karnatak University, Dharwad, India \\ Email: vasuki.arasi@yahoo.com
}

Received October 28, 2012; revised November 25, 2012; accepted December 3, 2012

\begin{abstract}
The crystal structure of the potential active N-[2-(6-Methoxy-2-oxo-2H-chromen-4-yl)-benzofuran-3-yl]-benzamide $\left(\mathrm{C}_{25} \mathrm{H}_{17} \mathrm{NO}_{5}\right)$ (I) has been determined from single crystal X-ray diffraction data. The title compound crystallizes in the monoclinic space group P 21/n, with $\mathrm{a}=12.0551(11), \mathrm{b}=9.7853(8), \mathrm{c}=16.6517(16) \AA, \beta=90.092(4)^{\circ}, \mathrm{V}=1964.28(3)$ $\AA^{3}, D_{\text {calc }}=1.391 \mathrm{Mg} / \mathrm{m}^{3}, \mathrm{Z}=4$. In the structure, intermolecular H-bonds lead to the formation of a centrosymmetric dimer of the molecule. There is an intramolecular $\mathrm{C} 7-\mathrm{H} 7 \ldots \mathrm{N} 1$ hydrogen bond forming a closed seven membered ring. There are also intramolecular $\pi-\pi$ interactions presented between the 3,6-Dihydro-2H-pyran ring of the chromen moiety $[\mathrm{Cg} 2 \ldots \mathrm{Cg} 2$ distance $=3.5812(13) \AA]$. The packing structure is stabilized by these $\mathrm{C}-\mathrm{H} \ldots \mathrm{N}, \mathrm{N}-\mathrm{H} \ldots \mathrm{O}$ hydrogen bonds, $\mathrm{C}-\mathrm{H} \ldots \pi$ and $\pi \ldots \pi$ interactions.
\end{abstract}

Keywords: Benzamide; Benzofuran; 2H-Chromen; Single Crystal Structure; X-Ray Diffraction

\section{Introduction}

The amide and sulfonamide moieties are the constituents of many biologically important compounds [1]. Coumarin derivatives are known to be an interesting class of natural or synthetic compounds, whose biological activity varies according to the substitutes on the benzopyran ring. Their antibacterial, antifungal, antitumor, anti-HIV and anti-inflammatory and analgesic activities have been published [2]. Benzamide derivatives, known for their anti-inflammatory and immunomodulatory, anti-tumoral, antipsychotic and antiallergic activities, are drugs widely used in medicine [3]. Benzanilides and benzamides exhibit wide range of biological activity and are extensively used in organic synthesis. Various N-substituted benzamides exhibit potent antiemetic activity [4]. The benzanilide core is presented in compounds with such a wide range of biological activities that it has been called a privileged structure. Benzanilides have established their efficacy as centroid elements of ligands that bind to a wide variety of receptor types. Thus benzanilides containing aminoalkyl groups originally designed as a peptidomimetic, have been incorporated in an Arg-Gly-Asp cyclic peptide yielding a high affinity GПIb/IIIa ligand. Imatinib is an ATP-site binding kinase inhibitor and

${ }^{*}$ Corresponding author. platelet-derived growth factor receptor kinases. Pyridylmethyl containing benzanilide are vascular endothelial growth factor receptor and tyrosine kinase inhibitor. Furthermore, benzamides have been reported to have activities as acetyl-CoA carboxylase and farnesyl transferase inhibitors [5]. N-substituted benzamides are well known anticancer compounds and the mechanism of action for $\mathrm{N}$-substituted benzamide-induced apoptosis has been studied, using declopramide as a lead compound. N-substituted benzamides inhibit the activity of nuclear factor$\mathrm{B}$ and nuclear factor of activated $\mathrm{T}$ cells activity while inducing activator protein 1 activity in $\mathrm{T}$ lymphocytes. Various $\mathrm{N}$-substituted benzamides exhibit potent antiemetic activity, while heterocyclic analogs of benzanilide derivatives are potassium channel activators. O-Aryloxylation of N-substituted benzamides induced by the copper (II)/trimethylamine $\mathrm{N}$-oxide system has been studied. N-Alkylated 2-nitrobenzamides are intermediates in the synthesis of dibenzo[b,e] $[1,4]$ diazepines and N-Acyl2-nitrobenzamides are precursors of 2,3-disubstitued $3 \mathrm{H}$ quinazoline-4-ones. A one-pot conversion of 2-nitro-narylbenzamides to 2,3-dihydro-1H-quinazoline-4-ones has also been reported [6]. As a part of studying the ring and side-chain substitutions on the crystal structures of chemically and biologically important class of compounds such as benzanilides, we report herein the crystal struc- 
ture of the title compound (I).

\section{Experimental}

\subsection{Synthesis of the Title Compound}

A suspension of 0.01 mole of 4-(3'-amino-2'-benzo (b) furanyl) coumarin and $8 \mathrm{ml}$ of benzoyl chloride was heated and stirred at $80^{\circ} \mathrm{C}$ for about 45 minutes. The mixture was cooled and solid separated was washed thoroughly with cold water and then with ethyl acetate to give sufficiently pure product. The compound is crystallised in alcohol, yield $93 \%$, melting point $204^{\circ} \mathrm{C}$. The compound is recrystallized from ethanol to get good quality single crystals.

\subsection{X-Ray Crystallography}

For the crystal structure determination, the single crystal of the compound $\mathrm{C}_{25} \mathrm{H}_{17} \mathrm{NO}_{5}$ was used for data collection on a Bruker Kappa APEXII CCD diffractometer [7]. The MoK $\alpha$ radiation of wavelength, $(\lambda=0.71073 \AA)$ and multi-scan technique for absorption correction were used for data collection. The lattice parameters were determined by the least-squares methods on the basis of all reflections with $\mathrm{F}^{2}>2 \sigma\left(\mathrm{F}^{2}\right)$. The structures were solved by direct methods using SHELXS-97 and refined by a full-matrix least-squares procedure using the program SHELXL-97 [8,9]. H atoms were positioned geometrically and refined using a riding model, fixing the aromatic $\mathrm{C}-\mathrm{H}$ distances at $0.93 \AA\left[\mathrm{U}_{\mathrm{iso}}(\mathrm{H})=1.2 \mathrm{U}_{\mathrm{eq}}(\mathrm{C})\right]$. The softwares used for Molecular graphics are ORTEP-3 for Windows [10] and PLATON [11]. The software used to prepare material for publication is WinGX publication routines [12]. Chemical structure of the title compound is shown in Figure 1. Molecular structure of the compound showing the atomic numbering scheme is shown in Figure 2(a). The crystallography details for the structures determination of the compound are presented in Table 1. Atomic coordinates and equivalent isotropic displacement parameters are shown in Table 2. Bond lengths and bond angles are shown in Table 3. Anisotropic displacement parameters are shown in Table 4. Hydrogen coordinates and isotropic displacement parameters are shown in Table 5. Torsion angles and Hydrogen bond geometry are shown in Tables 6 and 7 respectively.

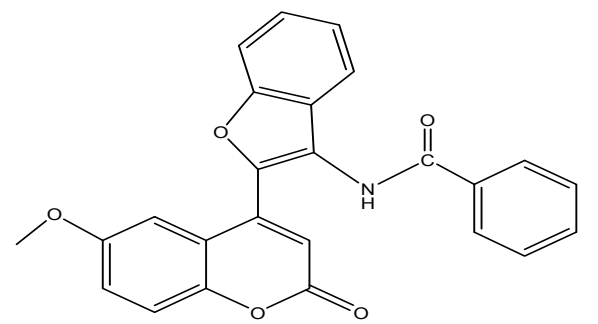

Figure 1. Chemical structure of the title compound.

\section{Results and Discussion}

Title compound crystallizes in the monoclinic centrosymmetric space group P21/n (No: 14 ) with $Z=4$. The structure of the compound consists of 6-Methoxy-2-oxo$2 \mathrm{H}$-chromen and Benzofuran fragments that connect to the benzamide moiety. None of the aromatic systems are coplanar with the benzamide unit. Dihedral angles between 1 - 2, 1 - 3 planes have been calculated. The mean plane through the benzamide unit $(1=\mathrm{N} 1 / \mathrm{C} 19 / \mathrm{O} 5 / \mathrm{C} 20$ $\mathrm{C} 25)$ is inclined at a dihedral angle of $65.85(5)^{\circ}$ with respect to the benzofuran unit $(2=\mathrm{O} 4 / \mathrm{C} 11-\mathrm{C} 18)$ and $79.36(5)^{\circ}$ with respect to the $2 \mathrm{H}$-chromen $(3=\mathrm{C} 2-\mathrm{C} 10 /$ $\mathrm{O} 2)$ ring system. The $\mathrm{C} 11-\mathrm{C} 12-\mathrm{N} 1$ angle is 126.86 $(19)^{\circ}$, implying that the benzofuran ring system attached at the $\mathrm{N} 1$ atom is pushed away from the benzamide unit. The relative orientation of this benzofuran system with

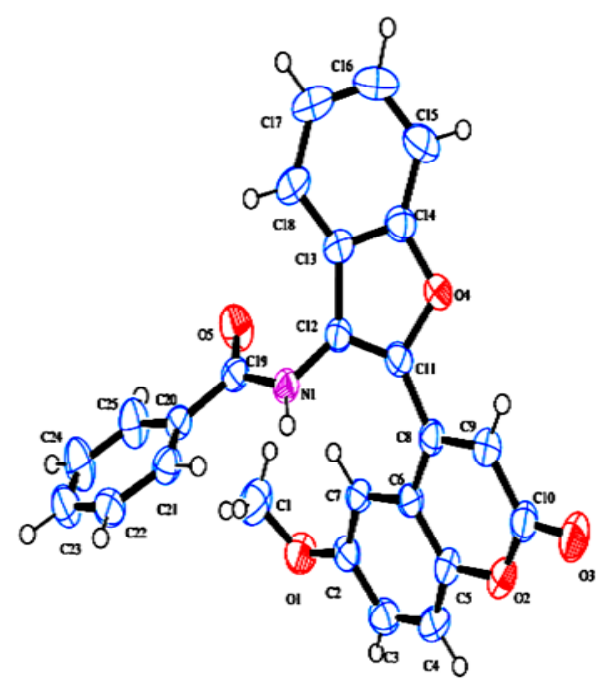

(a)

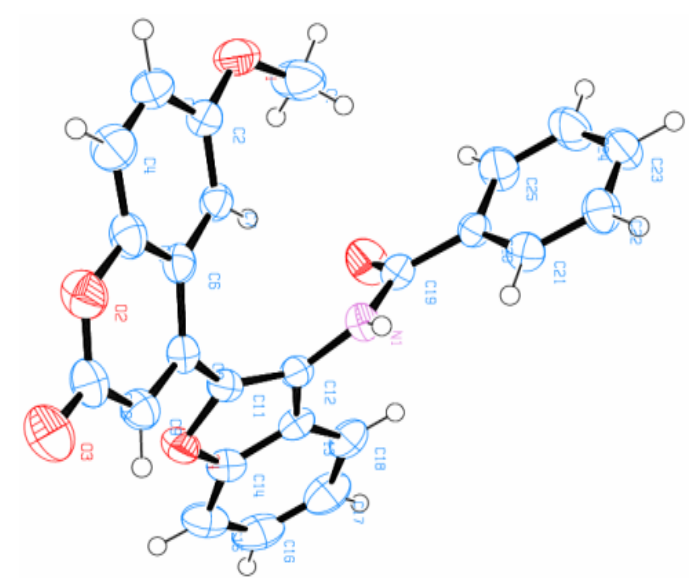

(b)

Figure 2. (a): Molecular structure of the title compound showing the atomic numbering system; (b): Molecular structure with different orientation of benzoyl group and benzofuran ring. Displacement ellipsoids are drawn at the $50 \%$ probability level. 
respect to the $2 \mathrm{H}$-chromen moiety is determined by the $\mathrm{C} 8-\mathrm{C} 11-\mathrm{C} 12-\mathrm{N} 1$ torsion angle $5.4(4)^{\circ}$, corresponding to $+\mathrm{sp}$.

The title compound shows an intramolecular $\mathrm{C}$ $\mathrm{H}$... N hydrogen bond (Table 7) which results in the formation of a seven membered ring $(\mathrm{N} 1-\mathrm{C} 12-\mathrm{C} 11-$ $\mathrm{C} 8-\mathrm{C} 6-\mathrm{C} 7-\mathrm{H} 7)$ and leads to a synperiplanar conformation between the benzofuran group and the $2 \mathrm{H}$-chromen group. The torsion angles in this region, $\mathrm{N} 1-\mathrm{C} 12-$ $\mathrm{C} 11-\mathrm{C} 8,5.4(4)^{\circ}$ and $\mathrm{C} 7-\mathrm{C} 6-\mathrm{C} 8-\mathrm{C} 11,-1.5(3)^{\circ}$ confirm this conformation. This formation forces the two amide hydrogen atoms to the opposite direction.

In the crystal structure, two intermolecular $\mathrm{N}-\mathrm{H}$...O $\left[\mathrm{N}(1) \ldots \mathrm{O}(3)^{\mathrm{a}}=3.003(3) \AA, \mathrm{N}(1)-\mathrm{H}(1) \ldots \mathrm{O}(3)^{\mathrm{a}}=\right.$ $156(2)^{\circ}$, symmetry code (a); 2-x,-y,-z] H-bonds, which lead to the formation of a centrosymmetric dimer of the molecule in the crystal unit cell (Figure 3). Here the molecules are linked by paired $\mathrm{N} 1-\mathrm{H} 1 \ldots \mathrm{O} 3$ hydrogen bonds in $\mathrm{R}_{2}^{2}$ (16) dimers, which are further linked into chains

Table 1. Crystal data and structure refinement.

\begin{tabular}{|c|c|c|}
\hline Temperature & 293(2) K & \\
\hline Wavelength & $0.71073 \AA$ & \\
\hline Crystal system & monoclinic & \\
\hline Space group & $\mathrm{P} 21 / \mathrm{n}$ & \\
\hline \multirow[t]{3}{*}{ Unit cell dimensions } & $\mathrm{a}=12.0551(11) \AA$ & $\alpha=90^{\circ}$ \\
\hline & $\mathrm{b}=9.7853(8) \AA$ & $\begin{array}{c}\beta=90.092 \\
(4)^{\circ}\end{array}$ \\
\hline & $c=16.6517(16) \AA$ & $\gamma=90^{\circ}$ \\
\hline Volume & $1964.3(3) \AA^{3}$ & \\
\hline $\mathrm{Z}$ & 4 & \\
\hline Density (calculated) & $1.391 \mathrm{Mg} / \mathrm{m}^{3}$ & \\
\hline Absorption coefficient & $0.098 \mathrm{~mm}^{-1}$ & \\
\hline $\mathrm{F}(000)$ & 856 & \\
\hline Crystal size & $0.20 \times 0.20 \times 0.20 \mathrm{~mm}^{3}$ & \\
\hline $\begin{array}{l}\text { Theta range for } \\
\text { data collection }\end{array}$ & $1.22^{\circ}$ to $24.99^{\circ}$ & \\
\hline Index ranges & $\begin{array}{l}-14<=\mathrm{h}<=14,-11<= \\
\mathrm{k}<=8,-19<=1<=19\end{array}$ & \\
\hline Reflections collected & 17190 & \\
\hline Independent reflections & $3455[\mathrm{R}($ int $)=0.0428]$ & \\
\hline $\begin{array}{l}\text { Completeness to theta }= \\
24.99^{\circ}\end{array}$ & $99.90 \%$ & \\
\hline Max. and min. transmission & 0.9807 and 0.9807 & \\
\hline Refinement method & $\begin{array}{l}\text { Full-matrix least-squares on } \\
\qquad \mathrm{F}^{2}\end{array}$ & \\
\hline Data/restraints/parameters & $3455 / 0 / 287$ & \\
\hline Goodness-of-fit on $\mathrm{F}^{2}$ & 1.038 & \\
\hline $\begin{array}{c}\text { Final } \mathrm{R} \text { indices }[\mathrm{I}> \\
2 \operatorname{sigma}(\mathrm{I})]\end{array}$ & $\mathrm{R} 1=0.0370, \mathrm{wR} 2=0.0845$ & \\
\hline $\mathrm{R}$ indices (all data) & $\mathrm{R} 1=0.0476, \mathrm{wR} 2=0.0915$ & \\
\hline Extinction coefficient & $0.0073(9)$ & \\
\hline Largest diff. peak and hole & 0.212 and $-0.152 \mathrm{e} \cdot \AA^{-3}$ & \\
\hline
\end{tabular}

Table 2. Atomic coordinates $\left(\times 10^{4}\right)$ and equivalent isotropic displacement parameters $\left(\AA^{2} \times 10^{3}\right)$. U(eq) is defined as one third of the trace of the orthogonalized $U^{\mathrm{ij}}$ tensor.

\begin{tabular}{ccccc}
\hline & $\mathbf{x}$ & $\mathbf{y}$ & $\mathbf{z}$ & $\mathbf{U}(\mathbf{e q})$ \\
\hline $\mathrm{O}(4)$ & $11144(1)$ & $2298(2)$ & $2014(1)$ & $40(1)$ \\
$\mathrm{O}(3)$ & $12148(2)$ & $-307(2)$ & $-446(1)$ & $60(1)$ \\
$\mathrm{O}(1)$ & $7940(2)$ & $5367(2)$ & $-523(1)$ & $53(1)$ \\
$\mathrm{N}(1)$ & $8170(2)$ & $2391(2)$ & $1750(1)$ & $34(1)$ \\
$\mathrm{C}(11)$ & $10195(2)$ & $2252(2)$ & $1543(1)$ & $34(1)$ \\
$\mathrm{C}(6)$ & $9883(2)$ & $2613(2)$ & $43(1)$ & $35(1)$ \\
$\mathrm{C}(9)$ & $11192(2)$ & $948(2)$ & $541(1)$ & $40(1)$ \\
$\mathrm{O}(2)$ & $11010(1)$ & $1284(2)$ & $-869(1)$ & $51(1)$ \\
$\mathrm{C}(12)$ & $9283(2)$ & $2508(2)$ & $1994(1)$ & $31(1)$ \\
$\mathrm{C}(22)$ & $4702(2)$ & $1585(2)$ & $1476(1)$ & $44(1)$ \\
$\mathrm{C}(8)$ & $10416(2)$ & $1898(2)$ & $704(1)$ & $33(1)$ \\
$\mathrm{C}(21)$ & $5822(2)$ & $1785(2)$ & $1598(1)$ & $38(1)$ \\
$\mathrm{C}(17)$ & $9826(3)$ & $3160(2)$ & $4199(1)$ & $56(1)$ \\
$\mathrm{C}(23)$ & $3985(2)$ & $2668(3)$ & $1497(2)$ & $48(1)$ \\
$\mathrm{C}(13)$ & $9665(2)$ & $2758(2)$ & $2800(1)$ & $34(1)$ \\
$\mathrm{C}(14)$ & $10800(2)$ & $2625(2)$ & $2776(1)$ & $39(1)$ \\
$\mathrm{C}(16)$ & $10973(3)$ & $3039(3)$ & $4152(2)$ & $59(1)$ \\
$\mathrm{C}(7)$ & $9102(2)$ & $3667(2)$ & $126(1)$ & $35(1)$ \\
$\mathrm{C}(24)$ & $4385(2)$ & $3955(3)$ & $1640(2)$ & $60(1)$ \\
$\mathrm{O}(5)$ & $7707(1)$ & $4463(2)$ & $2222(1)$ & $55(1)$ \\
$\mathrm{C}(20)$ & $6234(2)$ & $3079(2)$ & $1749(1)$ & $32(1)$ \\
$\mathrm{C}(15)$ & $11489(2)$ & $2776(3)$ & $3437(2)$ & $53(1)$ \\
$\mathrm{C}(2)$ & $8700(2)$ & $4331(2)$ & $-541(1)$ & $39(1)$ \\
$\mathrm{C}(5)$ & $10230(2)$ & $2292(2)$ & $-725(1)$ & $42(1)$ \\
$\mathrm{C}(18)$ & $9156(2)$ & $3031(2)$ & $3532(1)$ & $44(1)$ \\
$\mathrm{C}(10)$ & $11496(2)$ & $581(3)$ & $-263(2)$ & $44(1)$ \\
$\mathrm{C}(4)$ & $9847(2)$ & $2982(3)$ & $-1391(1)$ & $55(1)$ \\
$\mathrm{C}(3)$ & $9084(2)$ & $4005(3)$ & $-1305(1)$ & $51(1)$ \\
$\mathrm{C}(25)$ & $5493(2)$ & $4158(2)$ & $1772(2)$ & $51(1)$ \\
$\mathrm{C}(19)$ & $7416(2)$ & $3373(2)$ & $1930(1)$ & $34(1)$ \\
$\mathrm{C}(1)$ & $7353(2)$ & $5572(3)$ & $195(2)$ & $68(1)$ \\
\hline & & & & \\
\hline
\end{tabular}

by means of $\mathrm{C}-\mathrm{H} \ldots \pi$ (Table 7) and $\pi \ldots \pi$ stacking interactions. The latter involve the intramolecular interactions between 3,6-Dihydro-2H-pyran rings $(\mathrm{O} 2, \mathrm{C} 5, \mathrm{C} 6$, $\mathrm{C} 8, \mathrm{C} 9, \mathrm{C} 10=\mathrm{Cg} 2)$ of the chromen moiety $[\mathrm{Cg} 2 \ldots \mathrm{Cg} 2$ distance $=3.5812(13) \AA$; symmetry code: (i) $2-\mathrm{x},-\mathrm{y},-\mathrm{z}]$. Packing diagram and $\mathrm{H}$ bonding geometry along the a-axis [symmetry code: 2-x,-y,-z] is shown in Figure 4. $\mathrm{H}$ atoms involved in the interactions have only been shown in this Figure.

IR spectral details:

IR data of the title compound are: $3302(-\mathrm{NH}-), 1696(\mathrm{C}=\mathrm{O}), 1678(\mathrm{C}=\mathrm{O}), 1616(-\mathrm{C}=\mathrm{C})$, 1036 (-C-O-C-). 
Table 3. Bond lengths $[\AA ̊]$ and angles $\left[^{\circ}\right]$.

\begin{tabular}{|c|c|c|c|}
\hline $\mathrm{O}(4)-\mathrm{C}(14)$ & $1.375(3)$ & $\mathrm{C}(12)-\mathrm{C}(11)-\mathrm{C}(8)$ & $136.1(2)$ \\
\hline $\mathrm{O}(4)-\mathrm{C}(11)$ & $1.386(3)$ & $\mathrm{O}(4)-\mathrm{C}(11)-\mathrm{C}(8)$ & $113.33(18)$ \\
\hline $\mathrm{O}(3)-\mathrm{C}(10)$ & $1.211(3)$ & $\mathrm{C}(5)-\mathrm{C}(6)-\mathrm{C}(7)$ & $117.6(2)$ \\
\hline $\mathrm{O}(1)-\mathrm{C}(2)$ & $1.367(3)$ & $\mathrm{C}(5)-\mathrm{C}(6)-\mathrm{C}(8)$ & $117.2(2)$ \\
\hline $\mathrm{O}(1)-\mathrm{C}(1)$ & $1.404(3)$ & $\mathrm{C}(7)-\mathrm{C}(6)-\mathrm{C}(8)$ & $125.05(19)$ \\
\hline $\mathrm{N}(1)-\mathrm{C}(19)$ & $1.357(3)$ & $\mathrm{C}(8)-\mathrm{C}(9)-\mathrm{C}(10)$ & $122.6(2)$ \\
\hline $\mathrm{N}(1)-\mathrm{C}(12)$ & $1.406(3)$ & $\mathrm{C}(8)-\mathrm{C}(9)-\mathrm{H}(9)$ & 118.7 \\
\hline $\mathrm{N}(1)-\mathrm{H}(1)$ & $0.87(3)$ & $\mathrm{C}(10)-\mathrm{C}(9)-\mathrm{H}(9)$ & 118.7 \\
\hline $\mathrm{C}(11)-\mathrm{C}(12)$ & $1.355(3)$ & $\mathrm{C}(10)-\mathrm{O}(2)-\mathrm{C}(5)$ & $121.76(18)$ \\
\hline $\mathrm{C}(11)-\mathrm{C}(8)$ & $1.465(3)$ & $\mathrm{C}(11)-\mathrm{C}(12)-\mathrm{N}(1)$ & $126.86(19)$ \\
\hline $\mathrm{C}(6)-\mathrm{C}(5)$ & $1.381(3)$ & $\mathrm{C}(11)-\mathrm{C}(12)-\mathrm{C}(13)$ & $106.78(19)$ \\
\hline$C(6)-C(7)$ & $1.404(3)$ & $\mathrm{N}(1)-\mathrm{C}(12)-\mathrm{C}(13)$ & $125.96(19)$ \\
\hline$C(6)-C(8)$ & $1.453(3)$ & $\mathrm{C}(23)-\mathrm{C}(22)-\mathrm{C}(21)$ & $120.3(2)$ \\
\hline $\mathrm{C}(9)-\mathrm{C}(8)$ & $1.347(3)$ & $\mathrm{C}(23)-\mathrm{C}(22)-\mathrm{H}(22)$ & 119.8 \\
\hline$C(9)-C(10)$ & $1.434(3)$ & $\mathrm{C}(21)-\mathrm{C}(22)-\mathrm{H}(22)$ & 119.8 \\
\hline $\mathrm{C}(9)-\mathrm{H}(9)$ & 0.93 & $\mathrm{C}(9)-\mathrm{C}(8)-\mathrm{C}(6)$ & 119.1(2) \\
\hline $\mathrm{O}(2)-\mathrm{C}(10)$ & $1.353(3)$ & $\mathrm{C}(9)-\mathrm{C}(8)-\mathrm{C}(11)$ & $118.84(19)$ \\
\hline $\mathrm{O}(2)-\mathrm{C}(5)$ & $1.384(3)$ & $\mathrm{C}(6)-\mathrm{C}(8)-\mathrm{C}(11)$ & 121.91(19) \\
\hline $\mathrm{C}(12)-\mathrm{C}(13)$ & $1.439(3)$ & $\mathrm{C}(22)-\mathrm{C}(21)-\mathrm{C}(20)$ & $120.5(2)$ \\
\hline $\mathrm{C}(22)-\mathrm{C}(23)$ & $1.368(3)$ & $\mathrm{C}(22)-\mathrm{C}(21)-\mathrm{H}(21)$ & 119.8 \\
\hline $\mathrm{C}(22)-\mathrm{C}(21)$ & $1.380(3)$ & $\mathrm{C}(20)-\mathrm{C}(21)-\mathrm{H}(21)$ & 119.8 \\
\hline $\mathrm{C}(22)-\mathrm{H}(22)$ & 0.93 & $\mathrm{C}(18)-\mathrm{C}(17)-\mathrm{C}(16)$ & $121.9(2)$ \\
\hline$C(21)-C(20)$ & $1.384(3)$ & $\mathrm{C}(18)-\mathrm{C}(17)-\mathrm{H}(17)$ & 119.1 \\
\hline $\mathrm{C}(21)-\mathrm{H}(21)$ & 0.93 & $\mathrm{C}(16)-\mathrm{C}(17)-\mathrm{H}(17)$ & 119.1 \\
\hline $\mathrm{C}(17)-\mathrm{C}(18)$ & $1.378(4)$ & $\mathrm{C}(22)-\mathrm{C}(23)-\mathrm{C}(24)$ & $119.6(2)$ \\
\hline$C(17)-C(16)$ & $1.389(4)$ & $\mathrm{C}(22)-\mathrm{C}(23)-\mathrm{H}(23)$ & 120.2 \\
\hline $\mathrm{C}(17)-\mathrm{H}(17)$ & 0.93 & $\mathrm{C}(24)-\mathrm{C}(23)-\mathrm{H}(23)$ & 120.2 \\
\hline $\mathrm{C}(23)-\mathrm{C}(24)$ & $1.370(4)$ & $\mathrm{C}(14)-\mathrm{C}(13)-\mathrm{C}(18)$ & $118.9(2)$ \\
\hline $\mathrm{C}(23)-\mathrm{H}(23)$ & 0.93 & $\mathrm{C}(14)-\mathrm{C}(13)-\mathrm{C}(12)$ & $105.94(19)$ \\
\hline $\mathrm{C}(13)-\mathrm{C}(14)$ & $1.374(3)$ & $\mathrm{C}(18)-\mathrm{C}(13)-\mathrm{C}(12)$ & 135.1(2) \\
\hline $\mathrm{C}(13)-\mathrm{C}(18)$ & $1.393(3)$ & $\mathrm{C}(13)-\mathrm{C}(14)-\mathrm{O}(4)$ & $110.51(19)$ \\
\hline$C(14)-C(15)$ & $1.385(3)$ & $\mathrm{C}(13)-\mathrm{C}(14)-\mathrm{C}(15)$ & $124.3(2)$ \\
\hline $\mathrm{C}(16)-\mathrm{C}(15)$ & $1.368(4)$ & $\mathrm{O}(4)-\mathrm{C}(14)-\mathrm{C}(15)$ & $125.2(2)$ \\
\hline $\mathrm{C}(16)-\mathrm{H}(16)$ & 0.93 & $C(15)-C(16)-C(17)$ & $121.3(2)$ \\
\hline $\mathrm{C}(7)-\mathrm{C}(2)$ & $1.375(3)$ & $\mathrm{C}(15)-\mathrm{C}(16)-\mathrm{H}(16)$ & 119.3 \\
\hline $\mathrm{C}(7)-\mathrm{H}(7)$ & 0.93 & $\mathrm{C}(17)-\mathrm{C}(16)-\mathrm{H}(16)$ & 119.3 \\
\hline$C(24)-C(25)$ & $1.368(3)$ & $\mathrm{C}(2)-\mathrm{C}(7)-\mathrm{C}(6)$ & $120.2(2)$ \\
\hline $\mathrm{C}(24)-\mathrm{H}(24)$ & 0.93 & $\mathrm{C}(2)-\mathrm{C}(7)-\mathrm{H}(7)$ & 119.9 \\
\hline $\mathrm{O}(5)-\mathrm{C}(19)$ & $1.223(2)$ & $\mathrm{C}(6)-\mathrm{C}(7)-\mathrm{H}(7)$ & 119.9 \\
\hline$C(20)-C(25)$ & $1.383(3)$ & $\mathrm{C}(25)-\mathrm{C}(24)-\mathrm{C}(23)$ & $120.4(2)$ \\
\hline$C(20)-C(19)$ & $1.484(3)$ & $\mathrm{C}(25)-\mathrm{C}(24)-\mathrm{H}(24)$ & 119.8 \\
\hline $\mathrm{C}(15)-\mathrm{H}(15)$ & 0.93 & $\mathrm{C}(23)-\mathrm{C}(24)-\mathrm{H}(24)$ & 119.8 \\
\hline $\mathrm{C}(2)-\mathrm{C}(3)$ & $1.390(3)$ & $\mathrm{C}(25)-\mathrm{C}(20)-\mathrm{C}(21)$ & $118.2(2)$ \\
\hline $\mathrm{C}(5)-\mathrm{C}(4)$ & $1.378(3)$ & $\mathrm{C}(25)-\mathrm{C}(20)-\mathrm{C}(19)$ & $117.80(19)$ \\
\hline $\mathrm{C}(18)-\mathrm{H}(18)$ & 0.93 & $\mathrm{C}(21)-\mathrm{C}(20)-\mathrm{C}(19)$ & $123.92(19)$ \\
\hline $\mathrm{C}(4)-\mathrm{C}(3)$ & $1.368(4)$ & $\mathrm{C}(16)-\mathrm{C}(15)-\mathrm{C}(14)$ & $115.9(2)$ \\
\hline $\mathrm{C}(4)-\mathrm{H}(4)$ & 0.93 & $\mathrm{C}(16)-\mathrm{C}(15)-\mathrm{H}(15)$ & 122 \\
\hline $\mathrm{C}(3)-\mathrm{H}(3)$ & 0.93 & $\mathrm{C}(14)-\mathrm{C}(15)-\mathrm{H}(15)$ & 122 \\
\hline $\mathrm{C}(25)-\mathrm{H}(25)$ & 0.93 & $\mathrm{O}(1)-\mathrm{C}(2)-\mathrm{C}(7)$ & $124.6(2)$ \\
\hline $\mathrm{C}(1)-\mathrm{H}(1 \mathrm{~A})$ & 0.96 & $\mathrm{O}(1)-\mathrm{C}(2)-\mathrm{C}(3)$ & $114.5(2)$ \\
\hline $\mathrm{C}(1)-\mathrm{H}(1 \mathrm{~B})$ & 0.96 & $\mathrm{C}(7)-\mathrm{C}(2)-\mathrm{C}(3)$ & $120.9(2)$ \\
\hline $\mathrm{C}(1)-\mathrm{H}(1 \mathrm{C})$ & 0.96 & $C(4)-C(5)-C(6)$ & $122.2(2)$ \\
\hline $\mathrm{C}(14)-\mathrm{O}(4)-\mathrm{C}(11)$ & $106.20(17)$ & $\mathrm{C}(4)-\mathrm{C}(5)-\mathrm{O}(2)$ & $115.9(2)$ \\
\hline $\mathrm{C}(2)-\mathrm{O}(1)-\mathrm{C}(1)$ & $117.61(18)$ & $\mathrm{C}(6)-\mathrm{C}(5)-\mathrm{O}(2)$ & $121.9(2)$ \\
\hline $\mathrm{C}(19)-\mathrm{N}(1)-\mathrm{C}(12)$ & $121.23(19)$ & $\mathrm{C}(17)-\mathrm{C}(18)-\mathrm{C}(13)$ & $117.7(2)$ \\
\hline $\mathrm{C}(19)-\mathrm{N}(1)-\mathrm{H}(1)$ & $120.7(18)$ & $\mathrm{C}(17)-\mathrm{C}(18)-\mathrm{H}(18)$ & 121.2 \\
\hline $\mathrm{C}(12)-\mathrm{N}(1)-\mathrm{H}(1)$ & $118.0(18)$ & $\mathrm{C}(13)-\mathrm{C}(18)-\mathrm{H}(18)$ & 121.2 \\
\hline $\mathrm{C}(12)-\mathrm{C}(11)-\mathrm{O}(4)$ & $110.55(17)$ & $\mathrm{O}(3)-\mathrm{C}(10)-\mathrm{O}(2)$ & $117.3(2)$ \\
\hline $\mathrm{O}(2)-\mathrm{C}(10)-\mathrm{C}(9)$ & $117.2(2)$ & $\mathrm{O}(3)-\mathrm{C}(10)-\mathrm{C}(9)$ & $125.5(2)$ \\
\hline $\mathrm{C}(3)-\mathrm{C}(4)-\mathrm{C}(5)$ & $119.9(2)$ & $\mathrm{O}(5)-\mathrm{C}(19)-\mathrm{N}(1)$ & $120.9(2)$ \\
\hline $\mathrm{C}(3)-\mathrm{C}(4)-\mathrm{H}(4)$ & 120.1 & $\mathrm{O}(5)-\mathrm{C}(19)-\mathrm{C}(20)$ & $121.59(19)$ \\
\hline $\mathrm{C}(5)-\mathrm{C}(4)-\mathrm{H}(4)$ & 120.1 & $\mathrm{~N}(1)-\mathrm{C}(19)-\mathrm{C}(20)$ & $117.51(19)$ \\
\hline $\mathrm{C}(4)-\mathrm{C}(3)-\mathrm{C}(2)$ & $119.3(2)$ & $\mathrm{O}(1)-\mathrm{C}(1)-\mathrm{H}(1 \mathrm{~A})$ & 109.5 \\
\hline $\mathrm{C}(4)-\mathrm{C}(3)-\mathrm{H}(3)$ & 120.3 & $\mathrm{O}(1)-\mathrm{C}(1)-\mathrm{H}(1 \mathrm{~B})$ & 109.5 \\
\hline $\mathrm{C}(2)-\mathrm{C}(3)-\mathrm{H}(3)$ & 120.3 & $\mathrm{H}(1 \mathrm{~A})-\mathrm{C}(1)-\mathrm{H}(1 \mathrm{~B})$ & 109.5 \\
\hline$C(24)-C(25)-C(20)$ & $121.0(2)$ & $\mathrm{O}(1)-\mathrm{C}(1)-\mathrm{H}(1 \mathrm{C})$ & 109.5 \\
\hline $\mathrm{C}(24)-\mathrm{C}(25)-\mathrm{H}(25)$ & 119.5 & $\mathrm{H}(1 \mathrm{~A})-\mathrm{C}(1)-\mathrm{H}(1 \mathrm{C})$ & 109.5 \\
\hline $\mathrm{C}(20)-\mathrm{C}(25)-\mathrm{H}(25)$ & 119.5 & $\mathrm{H}(1 \mathrm{~B})-\mathrm{C}(1)-\mathrm{H}(1 \mathrm{C})$ & 109.5 \\
\hline
\end{tabular}

Table 4. Anisotropic displacement parameters $\left(\AA^{2} \times 10^{3}\right)$.

\begin{tabular}{|c|c|c|c|c|c|c|}
\hline & U11 & U22 & U33 & U23 & U13 & U12 \\
\hline $\mathrm{O}(4)$ & $34(1)$ & $49(1)$ & $37(1)$ & $-2(1)$ & $-6(1)$ & $6(1)$ \\
\hline $\mathrm{O}(3)$ & $52(1)$ & $63(1)$ & $66(1)$ & $-17(1)$ & $12(1)$ & $14(1)$ \\
\hline $\mathrm{O}(1)$ & $57(1)$ & $57(1)$ & $45(1)$ & $13(1)$ & $0(1)$ & $12(1)$ \\
\hline $\mathrm{N}(1)$ & $32(1)$ & $34(1)$ & $36(1)$ & $-7(1)$ & 2(1) & $0(1)$ \\
\hline $\mathrm{C}(11)$ & $34(1)$ & $34(1)$ & $34(1)$ & $2(1)$ & $-4(1)$ & 2(1) \\
\hline $\mathrm{C}(6)$ & $30(1)$ & $40(1)$ & $34(1)$ & $-2(1)$ & $2(1)$ & $-7(1)$ \\
\hline $\mathrm{C}(9)$ & $37(1)$ & $43(1)$ & $41(1)$ & $-3(1)$ & $2(1)$ & $2(1)$ \\
\hline $\mathrm{O}(2)$ & $50(1)$ & $63(1)$ & $41(1)$ & $-10(1)$ & $8(1)$ & $12(1)$ \\
\hline$C(12)$ & $32(1)$ & $31(1)$ & $31(1)$ & $2(1)$ & 1(1) & $2(1)$ \\
\hline$C(22)$ & $40(1)$ & $46(1)$ & $46(1)$ & $-9(1)$ & $-1(1)$ & $-7(1)$ \\
\hline $\mathrm{C}(8)$ & $29(1)$ & $34(1)$ & $37(1)$ & $-2(1)$ & $4(1)$ & $-2(1)$ \\
\hline $\mathrm{C}(21)$ & $40(1)$ & $34(1)$ & $39(1)$ & $-5(1)$ & $4(1)$ & $4(1)$ \\
\hline$C(17)$ & $86(2)$ & $52(2)$ & $29(1)$ & $-2(1)$ & $1(1)$ & $-9(2)$ \\
\hline $\mathrm{C}(23)$ & $33(1)$ & $59(2)$ & $53(2)$ & $2(1)$ & $-4(1)$ & $1(1)$ \\
\hline$C(13)$ & $45(1)$ & $28(1)$ & $31(1)$ & $3(1)$ & 1(1) & $-2(1)$ \\
\hline$C(14)$ & $44(2)$ & $35(1)$ & $36(1)$ & $2(1)$ & $-5(1)$ & $3(1)$ \\
\hline$C(16)$ & $87(2)$ & $50(2)$ & $38(1)$ & $4(1)$ & $-22(2)$ & $-5(2)$ \\
\hline $\mathrm{C}(7)$ & $34(1)$ & $42(1)$ & $29(1)$ & $2(1)$ & $3(1)$ & $-4(1)$ \\
\hline$C(24)$ & $40(2)$ & $47(2)$ & $91(2)$ & $4(2)$ & $-3(1)$ & $13(1)$ \\
\hline $\mathrm{O}(5)$ & $48(1)$ & $37(1)$ & $80(1)$ & $-16(1)$ & $-7(1)$ & $-2(1)$ \\
\hline $\mathrm{C}(20)$ & $33(1)$ & $34(1)$ & $30(1)$ & $0(1)$ & $4(1)$ & $-1(1)$ \\
\hline$C(15)$ & $56(2)$ & $52(2)$ & $51(2)$ & $2(1)$ & $-19(1)$ & $6(1)$ \\
\hline $\mathrm{C}(2)$ & $36(1)$ & $42(1)$ & $38(1)$ & $5(1)$ & $0(1)$ & $-3(1)$ \\
\hline $\mathrm{C}(5)$ & $35(1)$ & $52(1)$ & $39(1)$ & $-8(1)$ & $7(1)$ & 1(1) \\
\hline$C(18)$ & $58(2)$ & $42(1)$ & $32(1)$ & 1(1) & $7(1)$ & $-4(1)$ \\
\hline$C(10)$ & $35(1)$ & $48(1)$ & $49(1)$ & $-11(1)$ & $6(1)$ & $0(1)$ \\
\hline $\mathrm{C}(4)$ & $54(2)$ & $82(2)$ & $30(1)$ & $-1(1)$ & $4(1)$ & $4(2)$ \\
\hline $\mathrm{C}(3)$ & $49(2)$ & $69(2)$ & $34(1)$ & $10(1)$ & $-2(1)$ & $-2(1)$ \\
\hline$C(25)$ & $44(2)$ & $32(1)$ & $76(2)$ & $-3(1)$ & $5(1)$ & $5(1)$ \\
\hline$C(19)$ & $37(1)$ & $32(1)$ & $33(1)$ & $0(1)$ & $4(1)$ & $-2(1)$ \\
\hline $\mathrm{C}(1)$ & $73(2)$ & $77(2)$ & $53(2)$ & $8(1)$ & $7(2)$ & $35(2)$ \\
\hline
\end{tabular}

Table 5. Hydrogen coordinates $\left(\times 10^{4}\right)$ and isotropic displacement parameters $\left(\AA^{2} \times 10^{3}\right)$.

\begin{tabular}{ccccc}
\hline & $x$ & $y$ & $z$ & $U(e q)$ \\
\hline$H(9)$ & 11545 & 513 & 967 & 48 \\
$H(22)$ & 4432 & 709 & 1378 & 53 \\
$\mathrm{H}(21)$ & 6305 & 1044 & 1579 & 45 \\
$\mathrm{H}(17)$ & 9502 & 3334 & 4696 & 67 \\
$\mathrm{H}(23)$ & 3230 & 2531 & 1415 & 58 \\
$\mathrm{H}(16)$ & 11398 & 3138 & 4614 & 70 \\
$\mathrm{H}(7)$ & 8856 & 3917 & 634 & 42 \\
$\mathrm{H}(24)$ & 3901 & 4695 & 1648 & 71 \\
$\mathrm{H}(15)$ & 12256 & 2704 & 3397 & 64 \\
$\mathrm{H}(18)$ & 8390 & 3124 & 3570 & 53 \\
$\mathrm{H}(4)$ & 10107 & 2752 & -1899 & 66 \\
$\mathrm{H}(3)$ & 8824 & 4478 & -1751 & 61 \\
$\mathrm{H}(25)$ & 5753 & 5034 & 1879 & 61 \\
$\mathrm{H}(1 \mathrm{~A})$ & 6989 & 4738 & 346 & 101 \\
$\mathrm{H}(1 \mathrm{~B})$ & 6809 & 6276 & 117 & 101 \\
$\mathrm{H}(1 \mathrm{C})$ & 7859 & 5840 & 611 & 101 \\
$\mathrm{H}(1)$ & $7970(20)$ & $1660(30)$ & $1489(16)$ & $61(8)$ \\
\hline
\end{tabular}


Table 6. Torsion angles $\left[{ }^{\circ}\right]$.

\begin{tabular}{|c|c|}
\hline $\mathrm{C}(14)-\mathrm{O}(4)-\mathrm{C}(11)-\mathrm{C}(12)$ & $-1.4(2)$ \\
\hline $\mathrm{C}(14)-\mathrm{O}(4)-\mathrm{C}(11)-\mathrm{C}(8)$ & $-179.38(17)$ \\
\hline $\mathrm{O}(4)-\mathrm{C}(11)-\mathrm{C}(12)-\mathrm{N}(1)$ & $-171.91(19)$ \\
\hline $\mathrm{C}(8)-\mathrm{C}(11)-\mathrm{C}(12)-\mathrm{N}(1)$ & $5.4(4)$ \\
\hline $\mathrm{O}(4)-\mathrm{C}(11)-\mathrm{C}(12)-\mathrm{C}(13)$ & $1.1(2)$ \\
\hline $\mathrm{C}(8)-\mathrm{C}(11)-\mathrm{C}(12)-\mathrm{C}(13)$ & $178.4(2)$ \\
\hline $\mathrm{C}(19)-\mathrm{N}(1)-\mathrm{C}(12)-\mathrm{C}(11)$ & $-135.3(2)$ \\
\hline $\mathrm{C}(19)-\mathrm{N}(1)-\mathrm{C}(12)-\mathrm{C}(13)$ & $53.0(3)$ \\
\hline $\mathrm{C}(10)-\mathrm{C}(9)-\mathrm{C}(8)-\mathrm{C}(6)$ & $-1.8(3)$ \\
\hline $\mathrm{C}(10)-\mathrm{C}(9)-\mathrm{C}(8)-\mathrm{C}(11)$ & $-177.2(2)$ \\
\hline $\mathrm{C}(5)-\mathrm{C}(6)-\mathrm{C}(8)-\mathrm{C}(9)$ & $-1.6(3)$ \\
\hline$C(7)-C(6)-C(8)-C(9)$ & $-176.8(2)$ \\
\hline$C(5)-C(6)-C(8)-C(11)$ & $173.7(2)$ \\
\hline$C(7)-C(6)-C(8)-C(11)$ & $-1.5(3)$ \\
\hline $\mathrm{C}(12)-\mathrm{C}(11)-\mathrm{C}(8)-\mathrm{C}(9)$ & $-138.6(3)$ \\
\hline $\mathrm{O}(4)-\mathrm{C}(11)-\mathrm{C}(8)-\mathrm{C}(9)$ & $38.6(3)$ \\
\hline $\mathrm{C}(12)-\mathrm{C}(11)-\mathrm{C}(8)-\mathrm{C}(6)$ & $46.1(3)$ \\
\hline $\mathrm{O}(4)-\mathrm{C}(11)-\mathrm{C}(8)-\mathrm{C}(6)$ & $-136.68(19)$ \\
\hline $\mathrm{C}(23)-\mathrm{C}(22)-\mathrm{C}(21)-\mathrm{C}(20)$ & $0.5(4)$ \\
\hline $\mathrm{C}(21)-\mathrm{C}(22)-\mathrm{C}(23)-\mathrm{C}(24)$ & $0.0(4)$ \\
\hline $\mathrm{C}(11)-\mathrm{C}(12)-\mathrm{C}(13)-\mathrm{C}(14)$ & $-0.3(2)$ \\
\hline $\mathrm{N}(1)-\mathrm{C}(12)-\mathrm{C}(13)-\mathrm{C}(14)$ & $172.8(2)$ \\
\hline $\mathrm{C}(11)-\mathrm{C}(12)-\mathrm{C}(13)-\mathrm{C}(18)$ & $-177.7(2)$ \\
\hline $\mathrm{N}(1)-\mathrm{C}(12)-\mathrm{C}(13)-\mathrm{C}(18)$ & $-4.6(4)$ \\
\hline $\mathrm{C}(18)-\mathrm{C}(13)-\mathrm{C}(14)-\mathrm{O}(4)$ & $177.31(18)$ \\
\hline $\mathrm{C}(12)-\mathrm{C}(13)-\mathrm{C}(14)-\mathrm{O}(4)$ & $-0.6(2)$ \\
\hline $\mathrm{C}(18)-\mathrm{C}(13)-\mathrm{C}(14)-\mathrm{C}(15)$ & $-1.6(3)$ \\
\hline $\mathrm{C}(12)-\mathrm{C}(13)-\mathrm{C}(14)-\mathrm{C}(15)$ & $-179.4(2)$ \\
\hline $\mathrm{C}(11)-\mathrm{O}(4)-\mathrm{C}(14)-\mathrm{C}(13)$ & $1.2(2)$ \\
\hline $\mathrm{C}(11)-\mathrm{O}(4)-\mathrm{C}(14)-\mathrm{C}(15)$ & $-179.9(2)$ \\
\hline$C(18)-C(17)-C(16)-C(15)$ & $-0.4(4)$ \\
\hline$C(5)-C(6)-C(7)-C(2)$ & $0.8(3)$ \\
\hline $\mathrm{C}(8)-\mathrm{C}(6)-\mathrm{C}(7)-\mathrm{C}(2)$ & $176.0(2)$ \\
\hline $\mathrm{C}(22)-\mathrm{C}(23)-\mathrm{C}(24)-\mathrm{C}(25)$ & $-0.9(4)$ \\
\hline$C(22)-C(21)-C(20)-C(25)$ & $-0.1(3)$ \\
\hline$C(22)-C(21)-C(20)-C(19)$ & $176.6(2)$ \\
\hline $\mathrm{C}(17)-\mathrm{C}(16)-\mathrm{C}(15)-\mathrm{C}(14)$ & $-0.8(4)$ \\
\hline$C(13)-C(14)-C(15)-C(16)$ & $1.8(3)$ \\
\hline $\mathrm{O}(4)-\mathrm{C}(14)-\mathrm{C}(15)-\mathrm{C}(16)$ & $-176.9(2)$ \\
\hline $\mathrm{C}(1)-\mathrm{O}(1)-\mathrm{C}(2)-\mathrm{C}(7)$ & $-14.5(3)$ \\
\hline $\mathrm{C}(1)-\mathrm{O}(1)-\mathrm{C}(2)-\mathrm{C}(3)$ & $167.6(2)$ \\
\hline $\mathrm{C}(6)-\mathrm{C}(7)-\mathrm{C}(2)-\mathrm{O}(1)$ & $-180.0(2)$ \\
\hline $\mathrm{C}(6)-\mathrm{C}(7)-\mathrm{C}(2)-\mathrm{C}(3)$ & $-2.2(3)$ \\
\hline$C(7)-C(6)-C(5)-C(4)$ & $0.9(3)$ \\
\hline$C(8)-C(6)-C(5)-C(4)$ & $-174.7(2)$ \\
\hline $\mathrm{C}(7)-\mathrm{C}(6)-\mathrm{C}(5)-\mathrm{O}(2)$ & $179.0(2)$ \\
\hline $\mathrm{C}(8)-\mathrm{C}(6)-\mathrm{C}(5)-\mathrm{O}(2)$ & $3.4(3)$ \\
\hline $\mathrm{C}(10)-\mathrm{O}(2)-\mathrm{C}(5)-\mathrm{C}(4)$ & $176.5(2)$ \\
\hline $\mathrm{C}(10)-\mathrm{O}(2)-\mathrm{C}(5)-\mathrm{C}(6)$ & $-1.7(3)$ \\
\hline $\mathrm{C}(16)-\mathrm{C}(17)-\mathrm{C}(18)-\mathrm{C}(13)$ & $0.7(4)$ \\
\hline $\mathrm{C}(14)-\mathrm{C}(13)-\mathrm{C}(18)-\mathrm{C}(17)$ & $0.3(3)$ \\
\hline $\mathrm{C}(12)-\mathrm{C}(13)-\mathrm{C}(18)-\mathrm{C}(17)$ & $177.4(2)$ \\
\hline $\mathrm{C}(5)-\mathrm{O}(2)-\mathrm{C}(10)-\mathrm{O}(3)$ & $178.2(2)$ \\
\hline $\mathrm{C}(5)-\mathrm{O}(2)-\mathrm{C}(10)-\mathrm{C}(9)$ & $-1.7(3)$ \\
\hline $\mathrm{C}(8)-\mathrm{C}(9)-\mathrm{C}(10)-\mathrm{O}(3)$ & $-176.4(2)$ \\
\hline
\end{tabular}

\section{Continue}

\begin{tabular}{cc}
\hline $\mathrm{C}(8)-\mathrm{C}(9)-\mathrm{C}(10)-\mathrm{O}(2)$ & $3.5(3)$ \\
$\mathrm{C}(6)-\mathrm{C}(5)-\mathrm{C}(4)-\mathrm{C}(3)$ & $-1.1(4)$ \\
$\mathrm{O}(2)-\mathrm{C}(5)-\mathrm{C}(4)-\mathrm{C}(3)$ & $-179.3(2)$ \\
$\mathrm{C}(5)-\mathrm{C}(4)-\mathrm{C}(3)-\mathrm{C}(2)$ & $-0.3(4)$ \\
$\mathrm{O}(1)-\mathrm{C}(2)-\mathrm{C}(3)-\mathrm{C}(4)$ & $180.0(2)$ \\
$\mathrm{C}(7)-\mathrm{C}(2)-\mathrm{C}(3)-\mathrm{C}(4)$ & $2.0(4)$ \\
$\mathrm{C}(23)-\mathrm{C}(24)-\mathrm{C}(25)-\mathrm{C}(20)$ & $1.2(4)$ \\
$\mathrm{C}(21)-\mathrm{C}(20)-\mathrm{C}(25)-\mathrm{C}(24)$ & $-0.7(4)$ \\
$\mathrm{C}(19)-\mathrm{C}(20)-\mathrm{C}(25)-\mathrm{C}(24)$ & $-177.7(2)$ \\
$\mathrm{C}(12)-\mathrm{N}(1)-\mathrm{C}(19)-\mathrm{O}(5)$ & $9.1(3)$ \\
$\mathrm{C}(12)-\mathrm{N}(1)-\mathrm{C}(19)-\mathrm{C}(20)$ & $-171.69(18)$ \\
$\mathrm{C}(25)-\mathrm{C}(20)-\mathrm{C}(19)-\mathrm{O}(5)$ & $12.3(3)$ \\
$\mathrm{C}(21)-\mathrm{C}(20)-\mathrm{C}(19)-\mathrm{O}(5)$ & $-164.5(2)$ \\
$\mathrm{C}(25)-\mathrm{C}(20)-\mathrm{C}(19)-\mathrm{N}(1)$ & $-166.9(2)$ \\
$\mathrm{C}(21)-\mathrm{C}(20)-\mathrm{C}(19)-\mathrm{N}(1)$ & $16.3(3)$ \\
\hline
\end{tabular}

Table 7. Hydrogen bonds $\left[\AA\right.$ and $\left.{ }^{\circ}\right], \mathrm{Cg} 4$ is the centroid of the C13-C18 ring; $\mathrm{Cg} 5$ is the centroid of the C20-C25 ring.

\begin{tabular}{ccccc}
\hline $\mathrm{D}-\mathrm{H} \ldots \mathrm{A}$ & $\mathrm{d}(\mathrm{D}-\mathrm{H})$ & $\mathrm{d}(\mathrm{H} \ldots \mathrm{A})$ & $\mathrm{d}(\mathrm{D} \ldots \mathrm{A})$ & $<(\mathrm{DHA})$ \\
\hline $\mathrm{C}(7)-\mathrm{H}(7) \ldots \mathrm{N} 1$ & 0.93 & 2.52 & $3.184(3)$ & 128 \\
$\mathrm{~N}(1)-\mathrm{H}(1) \ldots \mathrm{O}(3)^{\mathrm{i}}$ & $0.87(3)$ & $2.19(3)$ & $3.003(3)$ & $156(2)$ \\
$\mathrm{C}(4)-\mathrm{H}(4) \ldots \mathrm{Cg}(5)^{\mathrm{ii}}$ & 0.93 & 2.54 & $3.427(3)$ & 160 \\
$\mathrm{C}(22)-\mathrm{H}(22) \ldots \mathrm{Cg}(4)^{\mathrm{iii}}$ & 0.93 & 2.78 & $3.608(3)$ & 149 \\
\hline
\end{tabular}

Note: Symmetry transformations used to generate equivalent atoms: (i) $1-\mathrm{x}+$ $2,-y,-z$; (ii) $1 / 2+\mathrm{x}, 1 / 2-\mathrm{y},-1 / 2+\mathrm{z}$; (iii) $3 / 2-\mathrm{x},-1 / 2+\mathrm{y}, 1 / 2-\mathrm{z}$.

The authenticity of the compound has been established from the obtained peak values.

\section{Conclusion}

The N-substituted benzamide derivatives have been reviewed for antibacterial, anti-inflammatory, analgesic and antiulcer actions. Pharmaceutical compositions of amide derivatives are used as, therapeutic agents for hypertension, angina, pectoris, asthma, renal and peripheral circulatory disturbances and inhibitors of vasospasm. The compounds are useful where cell death is due to trauma, viral infection, neurodegenerative disorder, cardiovascular disease, immune deficiency disorder, autoimmune disorder, renal disease, syndromes or pancreatitis. Substituted benzamides are acting as inhibitors of HIV protease [13]. These reported points are very useful to understand the benefit functions of the substituted benzamides in general. The title compound belongs to a class of 4-2'-benzofuranyl coumarins, the methoxy derivatives of which have exhibited potential anti inflammatory activity [14]. Further the 6-methoxy group is well known to undergo biotransformation to the corresponding 6-hydroxy coumarin possessing a phenolic group which enhances its ability of binding with biomolecules which is the basis of its pharmacological activity. The $\mathrm{N}$-benzoyl moiety will also undergo cleavage to generate 


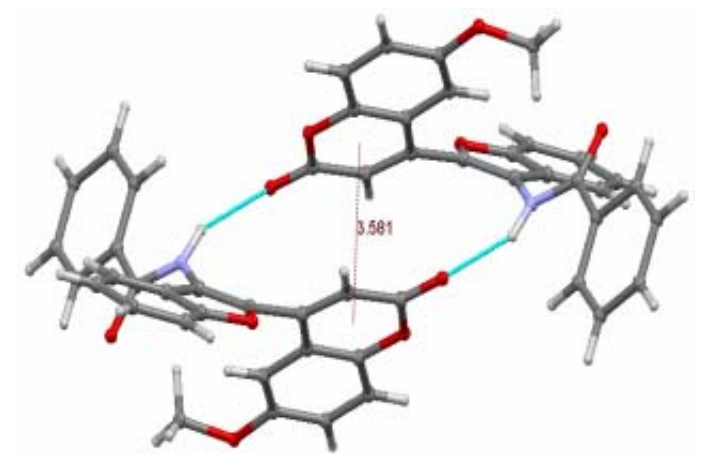

(a)

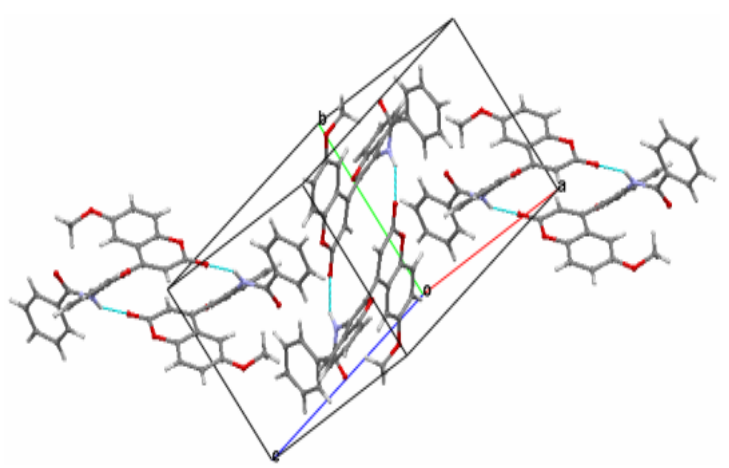

(b)

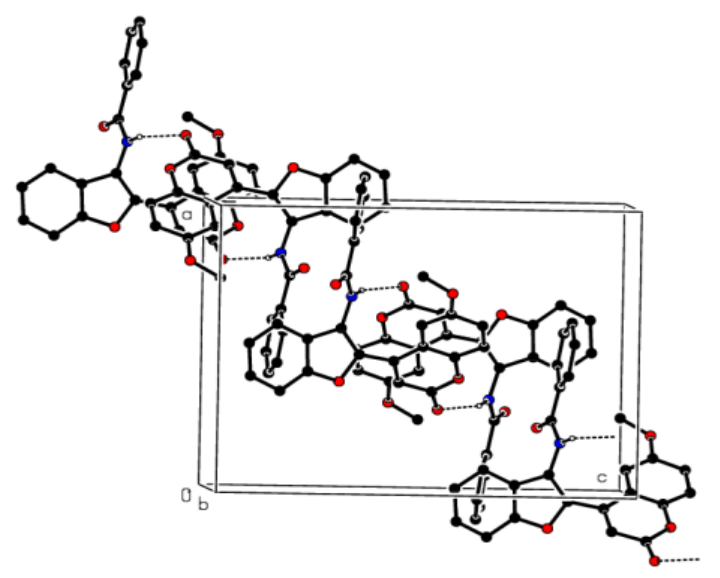

(c)

Figure 3. (a): Formation of centrosymmetric dimers; $\pi-\pi$ interaction shown as dotted line; (b): Part of the crystal structure of the compound, showing formation of a centrosymmetric $R_{2}{ }^{2}(16)$ dimmer; (c): Packing diagram, showing infinite chains running along the $b$-axis direction. Hydrogen bonds are shown as dashed lines. For the sake of clarity $H$ atoms not involved in the motif shown have been omitted.

a potential pharmacophoric amino group. The stereochemistry of the amide linkage has been established by present X-ray studies which is not possible by the conventional spectral studies. The title compound is air-stable in the solid state, crystallized from ethanol and insoluble in water. The authenticity of the compound has been established by IR technique. The crystallographic

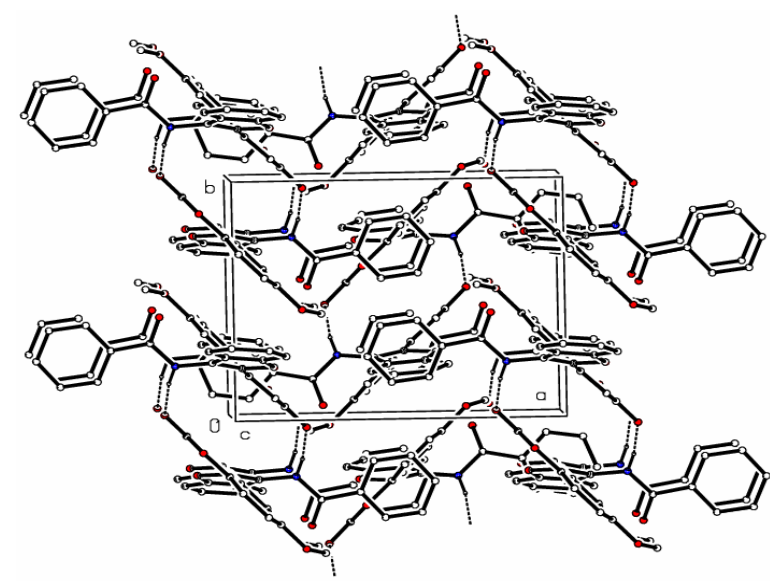

Figure 4. Packing diagram and $H$ bonding geometry along the a-axis [symmetry code: $2-x,-y,-z]$. $H$ atoms not involved in these interactions have been omitted for clarity.

investigation carried out was quite interesting by the zigzag packing of the dimers formed due to the two different orientations (Figures 2(a) and (b)) of the benzoyl group and the benzofuran ring. The amide was adopting the S-trans configuration and the packing diagram revealed a rare type of dimeric linkage between the lactone carbonyl and the amide $\mathrm{N}-\mathrm{H}$ bond. The compound characterized can be essential in medicinal and biological applications. The title structure may be important from a medicinal point of view as well as their widespread biological significance. The structure may be useful for further investigation on the mechanism, potential activity, optimal reaction condition etc.

\section{Acknowledgements}

The authors thank the Sophisticated Analytical Instrument Facility, IIT Madras, Chennai-36, for the data collection.

\section{REFERENCES}

[1] V. Z. Rodrigues, B. T. Gowda, V. Vrábel and J. Kožíšek, "4-Chloro-N-(3,5-Dimethylphenyl)Benzamide," Acta Crystallographica E, Vol. 68, Part 3, 2012, p. o820. doi:10.1107/S1600536812007180

[2] V. Maddi, S. N. Mamledesai, D. Satyanarayana and S. Swamy, "Synthesis and Antiinflammatory Activity of Substituted (2-Oxochromen-3-yl)Benzamides," Indian Journal of Pharamceutical Sciences, Vol. 69, No. 6, 2007, pp. 847-849. doi:10.4103/0250-474X.39452

[3] L. Muruganandam, S. Rajeswari, D. Tamilvendan, V. Ramkumar and G. V. Prabhu, "N-[Morpholino(phenyl)methyl] benzamide," Acta Crystallographica E, Vol. 65, Part 3, 2009, p. o578. doi:10.1107/S1600536809005327

[4] Anuradha Gurumoorthy, Vasuki Gopalsamy, K. Ramamurthi, Poonam Piplani and Ruchi Malik, "N-[4-(2-Morpholinoethoxy)Phenyl] Acetamide Monohydrate," Acta 
Crystllographica E, Vol. 67, Part 2, 2011, p. o262. doi:10.1107/S1600536810053675

[5] A. Saeed, S. Hussain and M. Bolte, "N-(2-MethoxyPhenyl)-2-Nitrobenzamide," Acta Crystallographica E, Vol. 64, Part 12, 2008, p. 0521. doi: $10.1107 /$ S1600536808002298

[6] A. Saeed, R. A. Khera, N. Abbas, J. Simpson and R. G. Stanley, "N-Butyl-4-Chlorobenzamide," Acta Crystallographica E, Vol. 64, Part 12, 2008, pp. o2322-o2323. doi:10.1107/S1600536808036313

[7] Bruker, "APEX2 and SAINT-Plus," Bruker AXS Inc., Madison, 2004.

[8] G. M. Sheldrick, "SHELXS-97 and SHELXL-97, Program for Crystal Structure Solution and Refinement," University of Gottingen, Gottingen, 1997.

[9] G. M. Sheldrick, "A Short History of SHELX," Acta Crystallographica A, Vol. 64, Part 1, 2008, pp. 112-122. doi:10.1107/S0108767307043930
[10] L. J. Farrugia, "ORTEP-3 for Windows-A Version of ORTEP-III with a Graphical User Interface (GUI)," Journal of Applied Crystallography, Vol. 30, No. 1, 1997, p. 565. doi:10.1107/S0021889897003117

[11] A. L. Spek, "Structure Validation in Chemical Crystallography," Acta Crystallographica D, Vol. 65, Part 2, 2009, pp. 148-155. doi:10.1107/S090744490804362X

[12] L. J. Farrugia, "WinGX Suite for Small-Molecule SingleCrystal Crystallography," Journal of Applied Crystallography, Vol. 32, Part 4, 1999, pp. 837-838. doi:10.1107/S0021889899006020

[13] Neerja Gupta and Ruby Naaz, International Journal of Pharma and Bio Sciences, Vol. 1, No. 3, 2010, pp. 1-7.

[14] M. D. Ghate, M. V. Kulkarni, R. Shobha and S. Y. Kattimani, "Synthesis of Vanillin Ethers from 4-(Bromomethyl) Coumarins as Anti-Inflammatory Agents," European Journal of Medicinal Chemistry, Vol. 38, No. 3, 2003, pp. 297-302. doi:10.1016/S0223-5234(03)00016-3 